\title{
Uma Comunidade de Prática para o ensino e a aprendizagem de programação
}

\author{
Vinicius Hartmann Ferreira - IFRS - vinihf@ gmail.com \\ Eliseo Berni Reategui - UFRGS - eliseoreategui@gmail.com
}

Resumo: As disciplinas de programação estão entre as disciplinas consideradas mais difíceis em cursos da área de Informática. O suporte da tecnologia ao ensino e a aprendizagem de programação tem apresentado resultados relevantes, sobretudo ao se valer dos aspectos sociais atrelados aos ambientes virtuais. Partindo deste princípio, este artigo apresenta a proposta de arquitetura de uma Comunidade de Prática, aliada aos recursos de formação de grupos homogêneos, a recomendação de tutores e a Modelagem Social Aberta do Estudante. Com base nos resultados de trabalhos similares entende-se que, possibilitar ao estudante com dificuldades de aprendizagem acompanhar e comparar o seu progresso com o dos demais em uma Comunidade de Prática, estimulando sua interação com outros participantes com diferentes níveis de conhecimento, possa auxiliá-lo a alcançar melhores resultados.

Palavras-chave: comunidade de prática, modelagem social aberta do estudante, ensino e aprendizagem de programação

\section{A Community of Practice for teaching and learning programming}

Abstract: Programming courses are considered as the most difficult in the IT area. The technology support to the process of teaching and learning programming has shown significant results, especially when they take advantage of the social aspects linked to virtual environments. This article presents the proposal for a Community of Practice, combined with features such as Open Social Student Modeling, the automatic formation of homogeneous groups and the recommendation of tutors. Based on the results achieved by similar work, give a student with learning disabilities the ability to monitor and compare your progress with the others in a community that encourages interaction between individuals of different levels of knowledge can help it to achieve better results.

Keywords: community of practice, open social student modeling, teaching and learning to program

\section{Introdução}

O ensino e a aprendizagem de algoritmos e de programação são tarefas complexas, tanto que disciplinas que envolvem estes conteúdos apresentam altas taxas de reprovação e são consideradas pelos estudantes como das mais difíceis nos cursos da área de Tecnologia da Informação e Comunicação (Watson e Li, 2014). Em um mapeamento sistemático da literatura realizado por Souza, Batista e Barbosa (2016) identificou-se que os principais problemas enfrentados no ensino e na aprendizagem de programação estão relacionados as dificuldades dos estudantes em compreender e aplicar os conceitos centrais e a sua falta de motivação para realizar as atividades propostas. Além disso, verificou-se que as principais soluções pesquisadas para estas dificuldades são a visualização de programas e algoritmos, o desenvolvimento de serious games e a criação de ambientes virtuais pedagógicos específicos.

V. $14 \mathrm{~N}^{\mathrm{o}} 2$, dezembro, 2016 
Iniciativas que buscam dar suporte à aprendizagem de programação através de meios que facilitem e estimulem interação entre os estudantes têm apresentado resultados relevantes, sobretudo quanto à motivação e à troca de informações entre estudantes com bom desempenho e aqueles que apresentam maior dificuldade de aprendizagem. Dentre estas iniciativas podem ser citadas as que envolvem a criação de Comunidades de Prática (Chen, Li e Wang, 2012) e as que possibilitam que os estudantes visualizem seu próprio rendimento e o comparem com o dos colegas ou da turma por meio da Modelagem Social Aberta do Estudante (Brusilovsky et al., 2015).

Partindo destes princípios, este artigo apresenta a proposta de criação de uma Comunidade de Prática enfocada no ensino e na aprendizagem de programação e incorporando a Modelagem Social Aberta do Estudante. Assim, o artigo está organizado de forma que os conceitos que fundamentam a proposta são apresentados nas seções 2 e 3 , a arquitetura da Comunidade de Prática é apresentada na seção 4 e as considerações finais na seção 5 .

\section{Comunidades de Prática}

O conceito de Comunidade de Prática (CdP) foi cunhado nos estudos de Lave e Wenger (1991) como uma forma de compreender o caráter social da aprendizagem humana, tendo como inspiração a teoria social e a antropologia. De acordo com Wenger (2010), a aprendizagem envolve relações sociais para além da relação mestre e aprendiz.

Uma CdP pode ser definida como um grupo de indivíduos que partilham interesse ou problemas a respeito de um determinado tópico e que por meio da interação mútua aprofundam seus conhecimentos nesta área (Wenger, Mcdermott e Snyder, 2002). Estas comunidades, que podem ser formais ou informais, estão em qualquer lugar, nas escolas, nos ambientes de trabalho ou mesmo em atividades cívicas, e um indivíduo pode se envolver com várias delas ao mesmo tempo. É importante destacar que a forma de participação em uma CdP pode variar conforme a expertise do indivíduo: em uma delas ele pode ser o centralizador das discussões enquanto em outras sua participação pode ser apenas periférica (Wenger, 1991).

Para a estruturação de uma CdP três fundamentos são essenciais: (i) o domínio: a comunidade necessita de um assunto/tópico para tratar; (ii) a comunidade: é prec iso um conjunto de indivíduos que interajam e cooperem na construção do conhecimento e esclarecimento de dúvidas; e (iii) a prática: o desenvolvimento de um repertório próprio dos membros a partir da prática compartilhada. Além disso, uma CdP deve envolver membros de três diferentes níveis: central, ativo e periférico, e estimular a interação entre eles. Inicialmente, um novo membro de uma CdP assume o nível periférico e a partir de suas interações e do aprofundamento de seu conhecimento pode passar para o nível ativo e posteriormente para o central, sendo esta mobilidade de níveis um papel importante destas comunidades (Wenger, Mcdermott e Snyder, 2002; Esteves et al., 2008).

Dado o crescente envolvimento da comunidade acadêmica com ambientes e redes virtuais, são encontrados na literatura diversos trabalhos sobre o uso de CdP na educação. $\mathrm{O}$ trabalho de Cordenonzi et al. (2013) apresenta um relato de criação de uma CdP focada na partilha de conhecimento sobre Mobile Learning. A comunidade que envolve pesquisadores, e que posteriormente passou a envolver estudantes, foi construída sobre a plataforma MOODLE, um Ambiente Virtual de Aprendizagem de código aberto. 
Schneider (2016) propõe a criação de uma CdP sobre práticas inclusivas e também analisa o perfil dos membros da comunidade. A partir do estudo desenvolvido, verificou-se que a CdP proposta constitui-se como um espaço de formação continuada e partilha de experiências a respeito do tema.

No trabalho de Chen, Li e Wang (2012) é proposta uma prática pedagógica na qual os estudantes são divididos em grupos para o desenvolvimento de atividades. Dentro dos grupos são definidos papeis que simulam um ambiente coorporativo, sendo estes: (i) gerente de projetos; (ii) líder de equipe; (iii) aprendiz; e (iv) programador. Conforme os estudantes demonstrassem evolução havia mobilidade de papeis. Ao final da atividade, identificou-se que foi positiva a iniciativa dado o número de estudantes que passaram de um papel periférico para um papel central na comunidade.

Esteves et al. (2008) propuseram uma CdP no mundo virtual Second Life para o ensino e a aprendizagem de algoritmos e programação. No trabalho, que relata os resultados de 2 anos do experimento, foi proposto aos estudantes que desenvolvessem projetos dentro do Second Life em duplas. Estas duplas poderiam interagir e trocar experiências dentro do mundo virtual para atingir o objetivo proposto. Verificou-se que os estudantes gostaram da prática, sobretudo pelo fato de o mundo virtual permitir que visualizassem de forma concreta o produto de sua codificação. Destaca-se ainda que, em virtude do Second Life ser um mundo virtual no qual cada indivíduo é representado por um avatar, as relações na $\mathrm{CdP}$ foram bastante frequentes.

Com relação a estes estudos, a proposta de utilização de CdP aqui descrita diferenciase principalmente com relação a sua área de aplicação (algoritmos e programação de computadores), mas também no que diz respeito ao emprego da Modelagem Social Aberta do Estudante, descrita na próxima seção.

\section{Modelagem Social Aberta do Estudante}

Com a crescente disponibilidade de plataformas sociais virtuais, motivar a aprendizagem por meio da interação com outros indivíduos tem sido um tema em evidência na educação mediada por tecnologia. Uma das formas de dar apoio aos estudantes nesse contexto, sem tirar com isso o seu senso de controle sobre o processo de aprendizagem, é o uso da Modelagem Aberta do Estudante (Open Student Modeling), a qual lhes permite observar e refletir sobre o seu progresso (Hsiao et al., 2011).

A Modelagem Aberta do Estudante (MAE) é uma abordagem oriunda da área de sistemas adaptativos. Para os pesquisadores desta área, permitir que o estudante visualize e até mesmo modifique o modelo gerado com base na sua interação com um sistema, na maioria das vezes encoberto e disponível apenas para o sistema personalizar as ações, é benéfico para a aprendizagem (Loboda et al., 2014).

Sistemas que trabalham com a MAE disponibilizam para os usuários o modelo de forma visual, podendo esta representação gráfica variar conforme o objetivo do sistema. Conforme pode ser visto na Figura 1, o usuário visualiza seu progresso em uma disciplina de programação Orientada a Objetos por meio de uma representação gráfica intitulada IntrospectiveViews (Hsiao et al., 2011). Dentre os principais projetos relacionados à MAE está seu uso como recurso para acesso a materiais, através do qual a MAE permite uma navegação personalizada e facilita ao estudante identificar os materiais que mais se adequam ao seu nível e às suas habilidades (Guerra et al., 2016). 


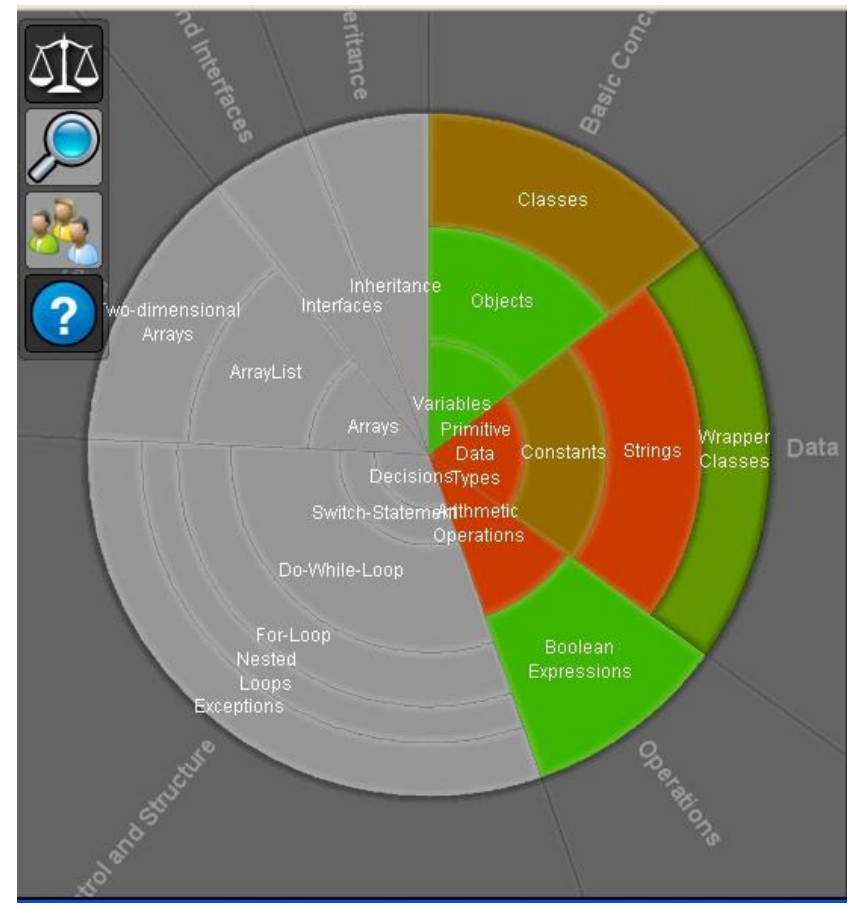

Figura 1 - Exemplo de visualização de Modelagem Aberta do Estudante. Fonte: Hsiao et al., 2011.

Uma outra proposta de uso da MAE é o seu compartilhamento com os demais membros da plataforma virtual, conhecido como Modelagem Social Aberta do Estudante (MSAE), ou do inglês Open Social Student Modeling. Esta ideia foi proposta com base na teoria da comparação social (Bull, 2007; Bull e Kay, 2007). A possibilidade de comparar o seu progresso com o de um colega, de um grupo específico ou de uma turma toda tem demonstrado aumentar a motivação para aprender do estudante e estimulado a sua participação em atividades acadêmicas (Loboda et al., 2014; Brusilovsky et al., 2015).

O trabalho de Hsiao et al. (2011) apresenta a incorporação da MSAE ao QuizJET, uma ferramenta para produção e disponibilização de questões parametrizadas acerca de programação em Java. A visualização da MSAE proposta neste trabalho se dá por meio de uma representação gráfica intitulada IntrospectiveViews, já apresentada na Figura 1. Conforme pode ser visto na Figura 2, durante a interação com a ferramenta o estudante pode acompanhar o seu progresso e compará-lo com o de outros colegas. No experimento relatado por Hsiao et al. (2011), que durou um semestre, verificou-se que os estudantes utilizaram o recurso tanto para acompanhar seu progresso quanto para navegar no ambiente virtual. Além disso, foi possível identificar maior sucesso nas respostas às questões quando comparadas com o sistema sem o recurso de IntrospectiveViews. 


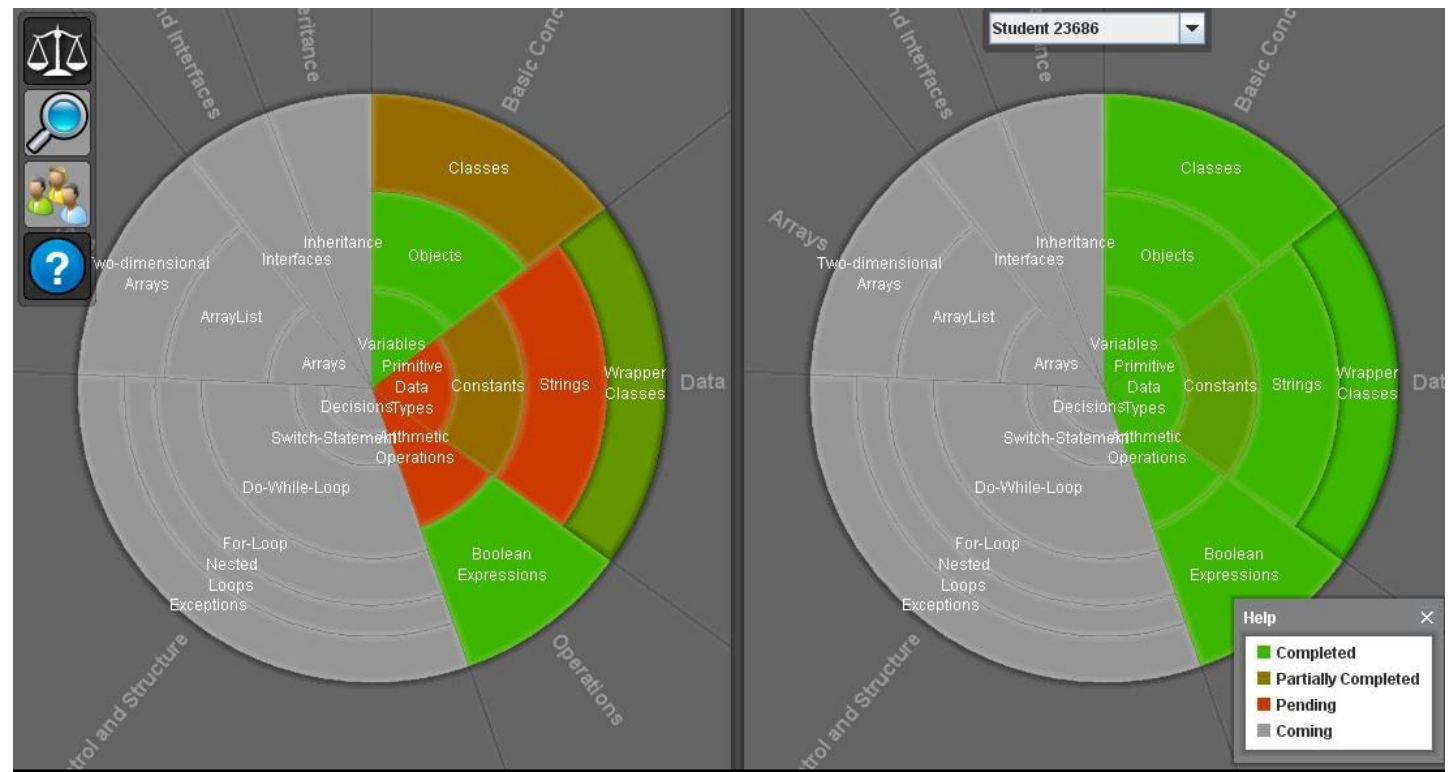

Figura 2 - Exemplo de visualização de Modelagem Social Aberta do Estudante. Fonte: Hsiao et al., 2011.

Os trabalhos de Loboda et al. (2014), Brusilovsky et al. (2015) e de Guerra et al. (2016) relatam experimentos com uma interface de código aberto para MSAE intitulada MasteryGrids. Esta interface permite visualizar o modelo do estudante, de seus colegas e de seus grupos e turmas. Conforme pode ser visto na Figura 3, as colunas da interface representam os tópicos e as linhas o progresso no tópico em questão. Ao clicar em uma célula, conforme pode ser visto na Figura 3, é possível visualizar em detalhes o desempenho em um tópico específico.

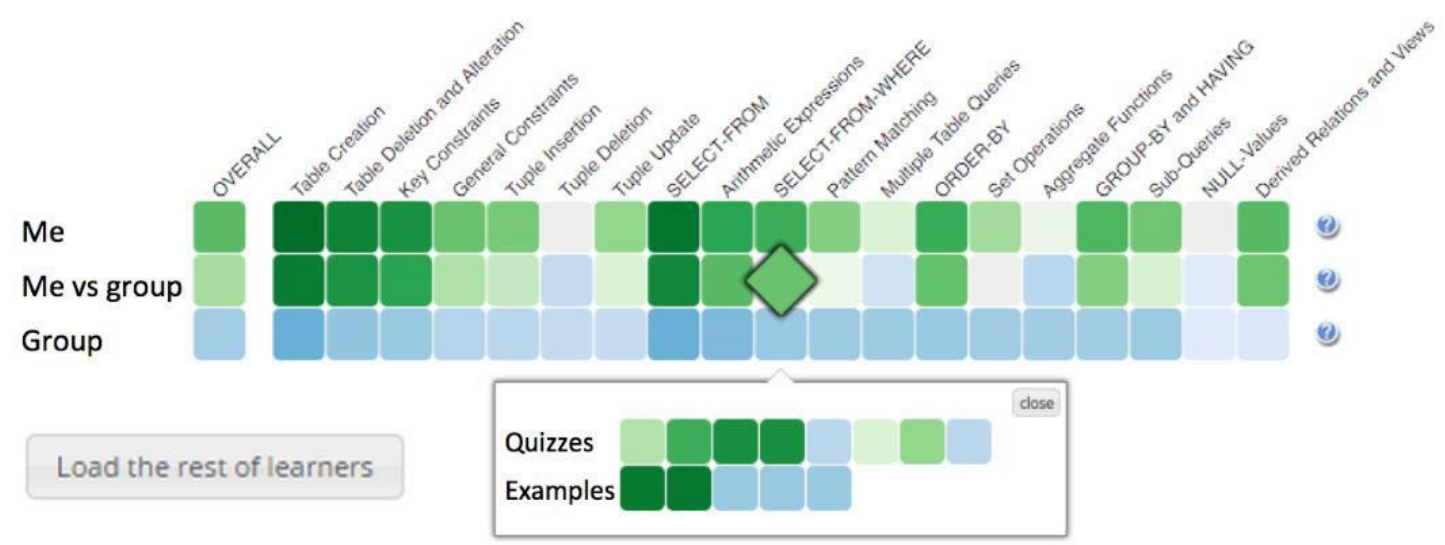

Figura 3 - Exemplo de visualização da MSAE na interface MasteryGrids. Fonte: Brusilovsky et al. (2015).

Ainda que os experimentos realizados com a interface MasteryGrids tenham abordado temas diferentes relacionados à computação, foi possível verificar em todos que os estudantes acessaram mais materiais quando comparado com interfaces diferentes. Além 
disso, como no experimento relatado por Hsiao et al. (2011), o número de acertos quando comparado com a MAE foi maior, sobretudo quando foi analisado o desempenho daqueles com mais dificuldades.

\section{Uma Comunidade de Prática para ensino e aprendizagem de programação}

Partindo do exposto até aqui, este artigo tem por objetivo apresentar a arquitetura de uma CdP focada no ensino e na aprendizagem de programação integrada à MSAE com o objetivo de estimular as interações entre os estudantes e prover recursos que deem suporte aos estudantes com dificuldades de aprendizagem. Sendo assim, inicialmente será descrita a visão geral da $\mathrm{CdP}$ e posteriormente os recursos de suporte, como a formação de grupos homogêneos e a recomendação de tutoria entre os membros da CdP.

\subsection{Visão geral da CdP}

Uma CdP fundamenta-se em três pilares, conforme já citado na seção 2, portanto é necessário inicialmente definir de que forma a CdP aqui proposta os atende:

- Domínio: o tópico central será algoritmos e programação, envolvendo desde princípios básicos da área até programação Web e Orientada a Objetos;

- Comunidade: estudantes da área de informática; e

- Prática: serão dispostos exercícios e desafios classificados por níveis de dificuldades e assuntos abordados, além de espaço para compartilhamento de novidades e descobertas.

Conforme pode ser visto na Figura 4, a CdP, construída sobre a plataforma MOODLE, integra recursos de comunicação assíncrona (fóruns, mensagens) e síncrona (chat), além de propor aos estudantes inscritos exercícios e desafios de programação. A interação do usuário com a CdP é registrada de forma que seja possível gerar para cada estudante uma MSAE. Este modelo apresenta informações sobre o progresso dos estudantes em cada assunto bem como seu grau de interação com os demais estudantes. Partindo disso, seguindo a ideia de uso da MSAE, os estudantes podem compartilhar o seu modelo com os demais estudantes e turmas da CdP bem como visualizar os modelos dos outros também. Conforme relatos de outros trabalhos, entende-se que conhecer o progresso dos colegas e compará-lo ao seu estimule os estudantes a participar mais das atividades e buscar maior sucesso. 


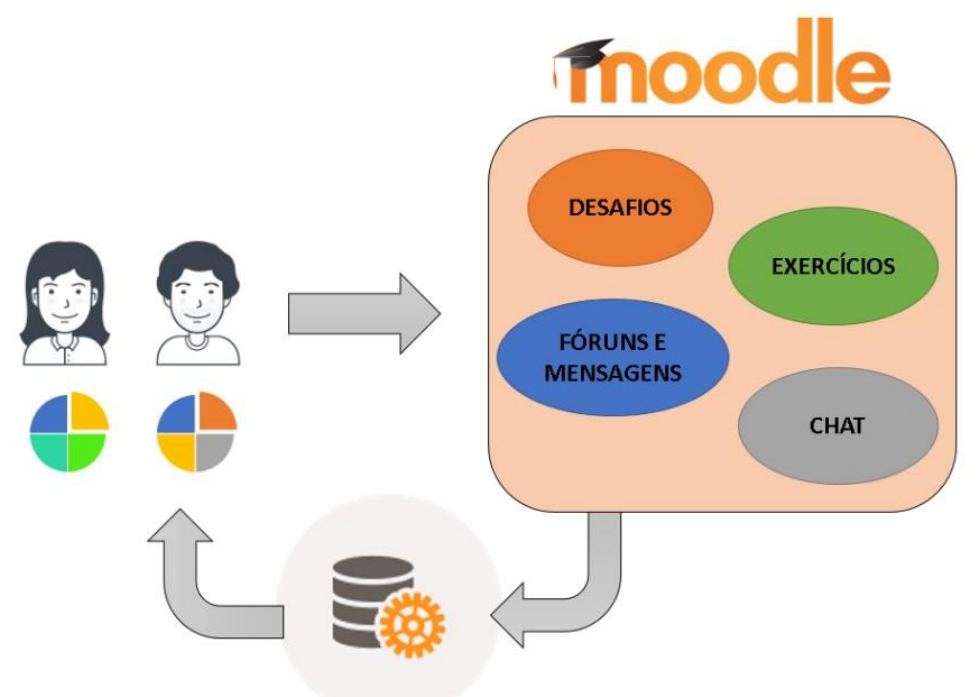

Figura 4 - Visão geral da Comunidade de Prática proposta.

\subsection{Formação de grupos}

Com o intuito de dar suporte a grupos específicos de estudantes com as mesmas dificuldades, são criados grupos dentro da CdP com base na MSAE dos estudantes de forma automática. Ao identificar estudantes com modelos de progresso similares são propostos grupos que colocam estes estudantes em contato. Desta forma entende-se que os estudantes podem construir o conhecimento com colegas que estejam em seu mesmo nível de desenvolvimento e também se sintam parte de uma comunidade. Dado que podem existir estudantes com dificuldades em diferentes assuntos propõe-se que um estudante possa participar de diferentes grupos ao mesmo tempo.

Trabalhos como os de Serrão et al. (2016) e de Testa e Marchi (2008) apresentam propostas de criação de grupos (redes sociais e sub comunidades respectivamente) com base no interesse dos membros de uma comunidade. O primeiro trabalho utiliza tags em postagens de fóruns para buscar indivíduos que estejam falando sobre o mesmo assunto e com isso cria uma rede social entre eles. $\mathrm{O}$ segundo trabalho utiliza um sistema multiagentes para agrupar indivíduos com interesses em comum.

Neste mesmo tema os trabalhos de Correia e Pimentel (2011) e de França e Amaral (2013) apresentam o uso de técnicas de mineração de dados para identificar estudantes com perfil similar e assim formar grupos para o ensino presencial. $\mathrm{O}$ primeiro trabalho tem foco na formação de turmas para recuperação paralela no ensino básico e o segundo tem por objetivo formar grupos de estudantes com dificuldades similares para atendimento de dificuldades no ensino de programação. Para a formação de grupos na CdP aqui proposta, seguiu-se a linha adotada por França e Amaral (2013), identificando por meio de técnicas de mineração de dados, sobretudo de clusterização, os estudantes com dificuldades similares a partir do seu desempenho nas atividades realizadas na CdP. 


\subsection{Recomendação de tutores}

Ao identificar estudantes com dificuldades e modelos de progresso similares e agrupá-los de forma homogênea, buscou-se promover a troca de informações entre os estudantes para construção coletiva de conhecimento, um dos objetivos centrais das CdP. Todavia, dado que uma comunidade que reúne estudantes com dificuldades similares possa ter problemas em definir estudantes para assumir o papel central, a CdP aqui apresentada propõe a recomendação de tutores, sendo estes estudantes com melhor desempenho. Para que seja respeitada a independência daquela comunidade gerada automaticamente uma recomendação deve ser aceita pela comunidade e só após este aceite o tutor pode integrá-la.

Pode-se citar como trabalho similar a esta proposta o trabalho de Reategui et al. (2006), que apresenta um agente de interface animado nomeado Kurrupako que, dentre outras atividades, recomenda tutores para os estudantes vinculados ao ambiente virtual A3. A recomendação feita pelo agente se dá levando em conta o estado de humor, as interações realizadas, o desempenho e a aceitação por parte dos outros participantes em relação às intervenções do estudante.

Para a recomendação dos tutores aos grupos formados na CdP aqui proposta, os mesmos critérios do agente Kurrupako foram empregados. Tendo em vista que das variáveis consideradas pelo agente para a recomendação de um tutor apenas o estado de humor não é nativo da plataforma MOODLE, torna-se simples a geração destas recomendações a partir da MSAE de cada estudante. O tutor neste caso é um membro do grupo que possui elevado potencial de assumir o papel central e auxiliar os colegas com dificuldades resolvendo dúvidas. Cada auxílio que o tutor dá a comunidade é avaliado, possibilitando a sugestão de novos tutores quando determinado estudante passa a não contribuir adequadamente no grupo.

\section{Considerações}

Este artigo apresentou a arquitetura de uma CdP com enfoque no ensino e na aprendizagem de algoritmos e programação. Esta CdP integra recursos que fomentam a interação entre usuários com bom desempenho e aqueles que enfrentam dificuldades de aprendizagem. Dentre os recursos implementados cita-se a MSAE para cada estudante, que permite o acompanhamento e comparação do progresso com os colegas, grupos e turmas, a criação de grupos que reúnam estudantes com dificuldades similares e a recomendação de colegas no papel de tutores para estes grupos.

Com base nos resultados dos trabalhos similares apresentados neste artigo, é possível afirmar que o emprego de uma CdP que possibilite aos estudantes acompanhar e comparar seu rendimento com o dos demais, possa estimular e auxiliar os estudantes na realização de atividades. Além disso, a partir da comparação e do auxílio de colegas com melhor desempenho, os estudantes podem ter acesso a recursos que os auxiliem a alcançar melhores resultados na aprendizagem de programação para além da sala de aula e da ajuda do professor.

Os próximos passos do projeto aqui descrito são a disponibilização da $\mathrm{CdP}$ para estudantes de disciplinas de algoritmos e de programação e o monitoramento de suas interações e de seu desempenho. Pretende-se com isso analisar e identificar como a participação em uma CdP, que dispõe dos recursos já descritos, contribui para a aprendizagem de estudantes que apresentam baixo rendimento em programação. 


\section{Referências bibliográficas}

BRUSILOVSKY, P.; SOMYÜREK, S.; GUERRA, J.; HOSSEINI, R.; ZADOROZHNY, V. The value of social: comparing open student modeling and open social student modeling. In: CONFERENCE ON USER MODELING, ADAPTATION AND PERSONALIZATION, 23., 2015, Dublin. Proceedings. Dublin: Springer, 2015.

BULL, S. UMPTEEN: Named and anonymous learner model access for instructors and peers. International Journal of Artificial Intelligence in Education, Berlin, v. 17, n. 3, p. 227-253. 2007.

BULL, S.; KAY, J. The SMILI (student models that invite the learner in): open learner modelling framework. International Journal of Artificial Intelligence in Education, Berlin, v. 17, n. 2, p. 89-120. 2007.

CHAGAS SCHNEIDER, F. Comunidade virtual de prática na perspectiva da inclusão escolar: o perfil, os discursos e as práticas de educadores no exercício da cultura da participação. Porto Alegre: PPGIE/UFRGS, 2016. 166 p. Tese de Doutorado.

CHEN, G. D.; LI, L. Y.; WANG, C. Y. A community of practice approach to learning programming. The Turkish Online Journal of Educational Technology, v. 22, n. 2, p. 1526. 2012.

CORDENONZI, W.; MÜLLER, T. J.; AMARAL, R. H.; PIOVESAN, S. D.; REATEGUI, E.; TAROUCO, L. M. R.; LIMA, J. V. Mobile Q: construção de uma comunidade de prática sobre mobile learning. RENOTE - Revista Novas Tecnologias na Educação, Porto Alegre, v. 11, n. 1, p. 1-10. 2013.

CORREIA, C. F.; PIMENTEL, E. P. Mineração de dados na formação de turmas para a recuperação paralela na educação básica. In: SIMPÓSIO BRASILEIRO DE INFORMÁTICA NA EDUCAÇÃO, 22., 2011, Aracaju. Anais. Aracaju: Sociedade Brasileira de Computação, 2011.

ESTEVES, M.; ANTUNES, R.; FONSECA, B.; MORGADO, L.; MARTINS, P. Using Second Life in programming's communities of practice. In: Groupware: Design, Implementation and Use. Berlin: Springer Berlin Heidelberg. 2008. p. 99-106.

FRANÇA, R. S.; AMARAL, H. J. C. Mineração de dados na identificação de grupos de estudantes com dificuldades de aprendizagem no ensino de programação. RENOTE Revista Novas Tecnologias na Educação, Porto Alegre, v. 11, n. 1, p. 1-10. 2013.

GUERRA, J.; SOMYUREK, S.; HOSSEINI, R.; BRUSILOVSKY, P. An intelligent interface for learning content: combining an open learner model and social comparison to support self-regulated learning and engagement. In: INTERNATIONAL CONFERENCE ON INTELLIGENT USER INTERFACES, 21., 2016, New York. Proceedings. New York: ACM, 2016. 
HSIAO, I. H.; BAKALOV, F.; BRUSILOVSKY, P.; KÖNIG-RIES, B. Open social student modeling: visualizing student models with parallel introspectiveviews. In: User Modeling, Adaption and Personalization. Berlin: Springer Berlin Heidelberg. 2011. p. 171-182.

LAVE, J.; WENGER, E. Situated learning: Legitimate Peripheral Participation. Cambridge: Cambridge University Press. 1991.

LOBODA, T. D.; GUERRA, J.; HOSSEINI, R.; BRUSILOVSKY, P. Mastery grids: an open source social educational progress visualization. In: Open Learning and Teaching in Educational Communities. Berlin: Springer International Publishing. 2014. p. 235-248.

REATEGUI, E.; CERON, R. F.; BOFF, E.; VICARI, R. M. Um agente animado para ambientes de aprendizagem colaborativos. Revista Brasileira de Informática na Educação, Porto Alegre, v. 14, n. 3, p. 27-38. 2006.

SERRÃO, R.; PINTO, S. C. C. S.; MONTEIRO, L.; CLUNIE, G. Construção automática de redes sociais móveis no ambiente Moodle. Revista Brasileira de Informática na Educação, Porto Alegre, v. 24, n. 1, p. 20-37. 2016.

SOUZA, D. M.; BATISTA, M. H. S.; BARBOSA, E. F. Problemas e dificuldades no ensino e na aprendizagem de programação: um mapeamento sistemático. Revista Brasileira de Informática na Educação, Porto Alegre, v. 24, n. 1, p. 39-52. 2016.

TESTA, C. D.; MARCHI, A. C. B. CV-Muzar - uma comunidade virtual utilizando tecnologias de IA para auxiliar na formação de sub-comunidades. RENOTE - Revista Novas Tecnologias na Educação, Porto Alegre, v. 6, n. 1, p. 1-10. 2008.

WATSON, C.; LI, F. W. B. Failure rates in introductory programming revisited. In: INNOVATION AND TECHNOLOGY IN COMPUTER SCIENCE EDUCATION (ITiCSE), 19., 2014, Uppsala, Sweden. Proceedings. New York: ACM, 2014.

WENGER, E.; MCDERMOTT, R.; SNYDER, W. Cultivating Communities of Practice. Boston: Harvard Business School Press. 2002. 